\title{
PARAMETRIC AMPLIFICATION OF RANDOM OPTICAL FIELDS
}

\author{
V. Pyragaitė and A. Stabinis \\ Department of Quantum Electronics, Vilnius University, Saulètekio 9, LT-10222 Vilnius, Lithuania \\ E-mail: viktorija.pyragaite@ff.vu.lt
}

Received 4 February 2009; revised 5 June 2009; accepted 18 June 2009

\begin{abstract}
We demonstrate that the initiated by quantum noise parametric downconversion of pump beam can be described in classical approach as a parametric amplification of orthogonal coaxial vortex modes with random amplitudes and of various topological charges $n$. It is shown that in the field of Gaussian pump beam the parametric gain is largest for the fundamental mode $(n=0)$. The evolution of correlation in the radial spectrum of different modes under parametric amplification is analysed.
\end{abstract}

Keywords: optical parametric amplifier, optical vortex

PACS: $42.65 . \mathrm{Yj}$, 42.60.Jf

\section{Introduction}

Starting from quantum noise optical parametric amplifier (OPA) emits a broadband spectrum of both temporal and spatial frequencies. Usually the coherence, which appears in this spectrum under parametric amplification by plane pump wave, is analysed by expansion of the random optical field in a large set of plane waves with random amplitudes and phases $[1,2]$. The finite diameter of pump beam leads to a coupling (correlation) of interacting plane waves $[1,3]$. An appearance of Bessel-like vortex beams from quantum noise in OPA with ring-shaped gain profile pumped by narrow Gaussian beam was demonstrated in Ref. [4]. The probability of a $J_{0}$-like beam increased with the decrease of pump beam diameter. $\mathrm{X}$-shaped spatiotemporal coherence can be observed in the random optical field amplified in OPA due to interplay of chromatic dispersion and diffraction [2,5-7]. The topological phase-defects are spontaneously generated from noise fluctuations in the degenerate OPA [8]. In the case of classical fields various phenomena of vortex amplification in OPA as well as parametric downconversion of optical vortices were investigated, see, e. g., [9-11]. The model of phase singularities (vortices) was also used to describe statistical properties of random fields (especially speckle) in linear optics, see [12-16].

In what follows, we show that the expansion of random optical field into a proper set of vortex modes with random amplitudes essentially simplifies the analysis of evolution of correlation in the optical field under prop- agation in OPA. We demonstrate that only two vortex modes with opposite topological charges are coupled in OPA. For this reason a large set of coupled differential equations describing the interaction of plane waves can be transferred into a rather small set of independent systems of two equations for each mode pair.

\section{Theoretical background}

Throughout the paper we consider a type $I$ degenerate OPA in which a pump beam (frequency $2 \omega$ ) is downconverted to a signal field (frequency $\omega$ ). For simplicity, our model is restricted to interaction of monochromatic waves, it neglects pump diffraction and depletion, and includes a classical description of the noise that initiates the process. In this case the equation of nonlinear optics for parametric amplification of diffracting random signal wave is

$$
\frac{\partial A}{\partial z}=-\frac{\mathrm{i}}{2 k_{s}}\left(\frac{\partial^{2}}{\partial x^{2}}+\frac{\partial^{2}}{\partial y^{2}}\right) A+\mathrm{i} \sigma A_{p} A^{*},
$$

where $A(x, y, z)$ is the complex amplitude of a random signal wave, $A_{p}(x, y)$ is the pump envelope, $x, y$ are transverse coordinates, $z$ is the direction of propagation, $k_{s}$ is the wave vector of signal wave, and $\sigma$ is the coupling coefficient in quadratic medium.

In cylindrical coordinates $(x=r \cos \psi, y=r \sin \psi)$ from Eq. (1) we have

$$
\frac{\partial}{\partial z} A(r, \psi, z)=-\frac{\mathrm{i}}{2 k_{s}}\left(\frac{1}{r} \frac{\partial}{\partial r} r \frac{\partial}{\partial r}+\frac{1}{r^{2}} \frac{\partial^{2}}{\partial \psi^{2}}\right) \times
$$




$$
\times A(r, \psi, z)+\mathrm{i} \sigma A_{p}(r) A^{*}(r, \psi, z),
$$

and it was assumed that the pump beam is axially symmetric. The signal field $A(r, \psi, z)$ can be represented as a superposition of components of an angular spectrum $S(\beta, \theta, z)$ :

$$
\begin{aligned}
& A(r, \psi, z)=\frac{1}{4 \pi^{2}} \\
& \times \int_{0}^{\infty} \int_{0}^{2 \pi} \beta S(\beta, \theta, z) \exp [-\mathrm{i} \beta r \cos (\theta-\psi)] \mathrm{d} \beta \mathrm{d} \theta,
\end{aligned}
$$

where $\beta$ is radial frequency. By use of Jacobi-Anger expansion

$$
\begin{aligned}
& \exp [-\mathrm{i} \beta r \cos (\theta-\psi)]= \\
& \sum_{n=-\infty}^{\infty}(-\mathrm{i})^{n} J_{n}(\beta r) \exp [\mathrm{i} n(\theta-\psi)]
\end{aligned}
$$

we obtain

$$
\begin{aligned}
& A(r, \varphi, z)=\frac{1}{2 \pi} \\
& \times \sum_{n=-\infty}^{\infty} \exp (-\mathrm{i} n \varphi) \int_{0}^{\infty} \beta S_{n}(\beta, z) J_{n}(\beta r) \mathrm{d} \beta,
\end{aligned}
$$

where

$$
S_{n}(\beta, z)=\frac{1}{2 \pi} \int_{0}^{2 \pi} S(\beta, \theta, z) \exp (\mathrm{i} n \theta) \mathrm{d} \theta
$$

is the $n$th azimuthal harmonic of angular spectrum $S(\beta, \theta, z), \varphi=\psi+\pi / 2$, and $J_{n}(\beta r)$ is the $n$ th-order Bessel function of the first kind. As a result, the complex amplitude of the signal field (Eq. (5)) has the form

$$
\begin{gathered}
A(r, \varphi, z)=\sum_{-\infty}^{\infty} A_{n}(r, \varphi, z), \\
A_{n}(r, \varphi, z)=F_{n}(r, z) \exp (-\mathrm{i} n \varphi),
\end{gathered}
$$

where

$$
F_{n}(r, z)=\frac{1}{2 \pi} \int_{0}^{\infty} \beta S_{n}(\beta, z) J_{n}(\beta r) \mathrm{d} \beta
$$

and $F_{n}(0, z)=0$ if $n \neq 0$. We note that integral $\int_{0}^{\infty} \int_{0}^{2 \pi} r A_{n} A_{m}^{*} \mathrm{~d} r \mathrm{~d} \varphi$ is zero for $m \neq n$. So, the signal field $A(r, \varphi, z)$ is represented as a superposition of orthogonal vortex modes $A_{n}(r, \varphi, z)$ with random amplitudes $F_{n}(r, z)$ and of various topological charges $n$. In Eq. (8) the quantity $S_{n}(\beta, z)$ is a radial spectrum of the $n$th mode. Further we suppose that at the input of OPA $(z=0)$ the components of angular spectrum $S(\beta, \theta)$ of the random field correspond to a Gaussian $\delta$-correlated noise such that

$$
\begin{aligned}
& \langle S(\beta, \theta)\rangle=0, \quad\left\langle S\left(\beta_{1}, \theta_{1}\right) S\left(\beta_{2}, \theta_{2}\right)\right\rangle=0, \\
& \left\langle S\left(\beta_{1}, \theta_{1}\right) S^{*}\left(\beta_{2}, \theta_{2}\right)\right\rangle=D \delta\left(\beta_{1}-\beta_{2}\right) \delta\left(\theta_{1}-\theta_{2}\right),(9)
\end{aligned}
$$

where parameter $D$ scales the noise level. In this case the components of the radial spectrum $S_{n}(\beta)$ at $z=0$ also correspond to a $\delta$-correlated noise,

$$
\begin{aligned}
& \left\langle S_{n}(\beta)\right\rangle=0, \quad\left\langle S_{n}\left(\beta_{1}\right) S_{n}\left(\beta_{2}\right)\right\rangle=0, \\
& \left\langle S_{n}\left(\beta_{1}\right) S_{n}^{*}\left(\beta_{2}\right)\right\rangle=\frac{D}{2 \pi} \delta\left(\beta_{1}-\beta_{2}\right),
\end{aligned}
$$

see Eq. (6), but the radial components of different vortex modes $m$ and $n$ are uncorrelated. Further we assume that the pump beam is Gaussian, $A_{p}=$ $a_{p} \exp \left(-r^{2} / d^{2}\right)$, substitute Eq. (7) into Eq. (2), and, as a result, for the $n$th mode we obtain

$$
\begin{aligned}
\frac{\partial F_{n}}{\partial z}= & -\frac{\mathrm{i}}{2 k_{s}}\left(\frac{1}{r} \frac{\partial}{\partial r} r \frac{\partial}{\partial r}-\frac{n^{2}}{r^{2}}\right) F_{n} \\
& +\mathrm{i} \Gamma \exp \left(-r^{2} / d^{2}\right) F_{-n}^{*},
\end{aligned}
$$

where $\Gamma=\sigma a_{p}$. We take into account that

$$
\left(\frac{1}{r} \frac{\partial}{\partial r} r \frac{\partial}{\partial r}-\frac{n^{2}}{r^{2}}\right) F_{n}=-\frac{1}{2 \pi} \int_{0}^{\infty} \beta^{3} S_{n}(\beta) J_{n}(\beta r) \mathrm{d} \beta,
$$

and rewrite Eq. (11) in the following form:

$$
\begin{aligned}
& \frac{\partial F_{n}}{\partial z}=\frac{\mathrm{i}}{4 \pi k_{s}} \int_{0}^{\infty} \gamma^{3} S_{n}(\gamma) J_{n}(\gamma r) \mathrm{d} \gamma \\
& +\mathrm{i} \Gamma \exp \left(-r^{2} / d^{2}\right) F_{-n}^{*} .
\end{aligned}
$$

An expansion of $F_{n}$ into a radial spectrum $S_{n}$, see Eq. (8), gives

$$
\frac{\partial S_{n}(\beta, z)}{\partial z}=\frac{\mathrm{i} \beta^{2}}{2 k_{s}} S_{n}(\beta, z)+\mathrm{i}(-1)^{n} \Gamma \frac{d^{2}}{2}
$$

$\times \int_{0}^{\infty} \gamma \exp \left[-\frac{\left(\beta^{2}+\gamma^{2}\right) d^{2}}{4}\right] I_{n}\left(\frac{\beta \gamma d^{2}}{2}\right) S_{-n}^{*}(\gamma, z) \mathrm{d} \gamma$, 
where $I_{n}$ is a modified Bessel function of $n$th order. The obtained Eq. (14) describes an evolution of the radial spectrum of $n$th signal vortex mode amplified in OPA, which is pumped by a Gaussian beam with a beamwidth $d$.

Further we include into consideration the normalized variables $\xi=z / l(0 \leq \xi \leq 1), p=\beta / \beta_{0}, q=\gamma / \beta_{0}$, where $l$ is the length of OPA crystal, $\beta_{0}^{2}=k_{p} \sqrt{2 \Gamma / l}$, and $k_{p} \approx 2 k_{s}$ is a wave vector of the pump beam. Then Eq. (14) can be written as

$$
\begin{aligned}
& \frac{\partial S_{n}(p, \xi)}{\partial \xi}=\mathrm{i} \sqrt{G} p^{2} S_{n}(p, \xi)+\mathrm{i}(-1)^{n} G \\
& \times \int_{0}^{\infty} q H_{n}(p, q) S_{-n}^{*}(q, \xi) \mathrm{d} q,
\end{aligned}
$$

where $G=2 \Gamma l$ is a parametric gain parameter,

$$
H_{n}(p, q)=\alpha \exp \left[-\left(p^{2}+q^{2}\right) \alpha\right] I_{n}(2 \alpha p q),
$$

here $\alpha=L_{d} \sqrt{G} /(2 l)$, and $L_{d}=k_{p} d^{2} / 2$ is a Rayleigh length of the pump beam. The correlations appearing in the radial spectrum $S_{n}$ under parametric amplification are caused by coupling function $H_{n}(p, q)$ which peaks at $p=q$. The dependence $H_{n}(p, p)$ on $p$ for two signal modes and two values of parameter $\alpha$ are shown in Fig. 1. The width of function $H_{n}(p, p)$ (dotted lines at 0.5 level for $H_{0}(p, p)$ ) as well as degree of correlation in the radial spectrum increase with a decrease of parameter $\alpha \propto d^{2}$, but parametric gain in this case is smaller because the power of the pump beam is proportional to $d^{2}$. We note that the coupling of radial components of signal vortex modes is rather weak in comparison with a fundamental mode $(n=0)$, Fig. 1 .

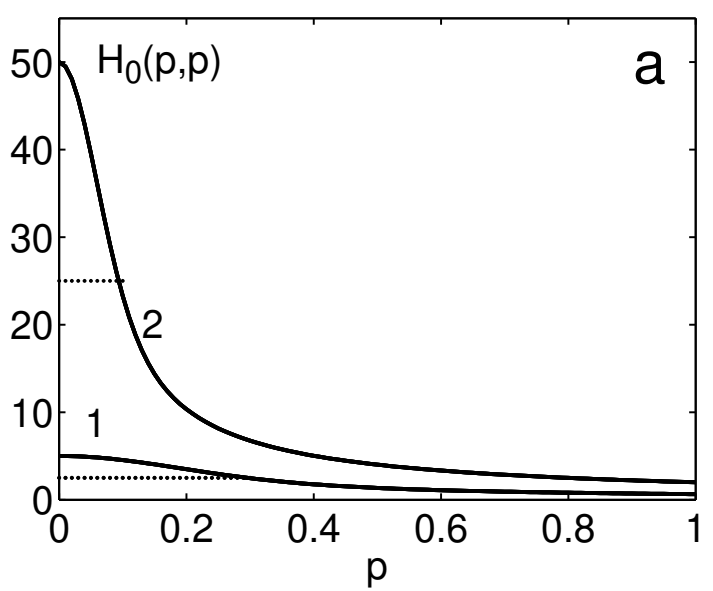

\section{Correlation functions of radial spectrum}

Further we consider two correlation functions $B_{1 n}\left(p_{1}, p_{2}\right)$ and $B_{2 n}\left(p_{1}, p_{2}\right)$ for radial spectrum of signal modes:

$$
\begin{aligned}
& B_{1 n}\left(p_{1}, p_{2}\right)=\left\langle S_{n}\left(p_{1}\right) S_{n}^{*}\left(p_{2}\right)\right\rangle, \\
& B_{2 n}\left(p_{1}, p_{2}\right)=\left\langle S_{n}\left(p_{1}\right) S_{-n}\left(p_{2}\right)\right\rangle .
\end{aligned}
$$

\subsection{Plane pump wave}

In the limit case of plane pump wave $(d \rightarrow \infty$, $\alpha \rightarrow \infty)$ the correlation functions $B_{1 n}\left(p_{1}, p_{2}\right)$ and $B_{2 n}\left(p_{1}, p_{2}\right)$ can be easily found. At $\alpha \rightarrow \infty$ Eq. (15) has a quite simple form:

$$
\frac{\partial S_{n}(p, \xi)}{\partial \xi}=\mathrm{i} \sqrt{G} p^{2} S_{n}(p, \xi)+\mathrm{i}(-1)^{n} \frac{G}{2} S_{-n}^{*}(p, \xi) .
$$

A solution of Eq. (18) is

$$
\begin{aligned}
& S_{n}(p, \xi)=\left[\cosh (\lambda \xi)+\mathrm{i} \frac{\sqrt{G}}{\lambda} p^{2} \sinh (\lambda \xi)\right] S_{n 0}(p) \\
& +\frac{\mathrm{i}(-1)^{n} G}{2 \lambda} \sinh (\lambda \xi) S_{-n 0}(p)^{*}
\end{aligned}
$$

where $\lambda=G \sqrt{1-4 p^{4} / G} / 2$, and $S_{n 0}(p)$ is a radial spectrum of $n$th mode at the input of OPA. It is obvious that components of radial spectrum are uncorrelated.

Using Eq. (19) for correlation function $B_{1 n}\left(p_{1}, p_{2}\right)$ we obtain that

$B_{1 n}\left(p_{1}, p_{2}\right)=\frac{D G}{8 \pi \lambda_{1}^{2}}\left[G \cosh \left(2 \lambda_{1} \xi\right)-4 p_{1}^{4}\right] \delta\left(p_{1}-p_{2}\right)$,

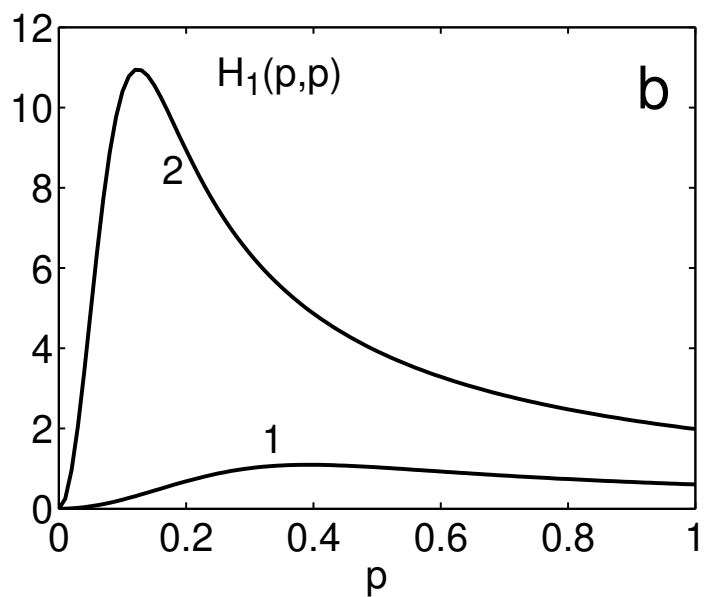

Fig. 1. Dependence of coupling function $H_{n}(p, p)$ on radial frequency $p$ for two signal modes: (a) $n=0$, (b) $n=1 . \alpha: 5$ (1), 50 (2). 
where $\lambda_{1}=\lambda\left(p_{1}\right)$. The spectral intensity $\left\langle\left|S_{n}(p)\right|^{2}\right\rangle=$ $B_{1 n}(p, p)$ for large gain $G \xi \gg 1$ is

$$
\left\langle\left|S_{n}(p)\right|^{2}\right\rangle \approx \frac{D}{4 \pi} \exp \left[\left(G-2 p^{4}\right) \xi\right] .
$$

The parametric gain of the component $p$ depends on its value due to diffraction but does not depend on the mode number. So, the parametric gain as well as the bandwidth of radial frequencies are the same for all modes. In this case an intensity of $n$th mode at the output of OPA $(\xi=1)$ for $G \gg 1$ is

$$
\left\langle\left|F_{n}(\rho)\right|^{2}\right\rangle=\frac{\beta_{0}^{4} D}{16 \pi^{3}} \exp (G) \int_{0}^{\infty} p^{2} \exp \left(-2 p^{4}\right) J_{n}^{2}(p \rho) \mathrm{d} p,
$$

where $\rho=\beta_{0} r$. The distribution of normalized intensity $P_{n}(\rho)=\left\langle\left|F_{n}(\rho)\right|^{2}\right\rangle /\left\langle\left|F_{0}(0)\right|^{2}\right\rangle$ for three signal modes is shown in Fig. 2. We note that under paramet-

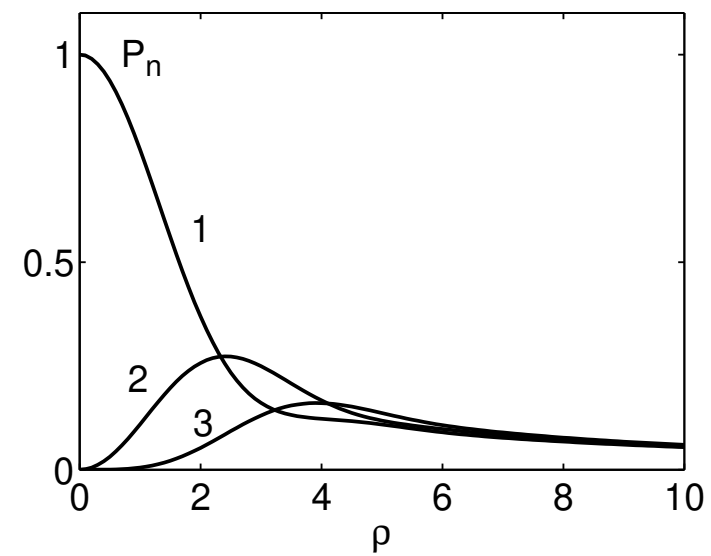

Fig. 2. Distribution of normalized intensity of signal modes at the output of OPA. Large parametric gain, plane pump wave. $n$ : 0 (1), $1(2), 2(3)$.

ric amplification the correlation between signal modes with opposite topological charges appears. At large parametric gain $G \xi \gg 1$ we find

$$
B_{2 n}\left(p_{1}, p_{2}\right) \approx \mathrm{i} B_{1 n}\left(p_{1}, p_{2}\right) \text {. }
$$

\subsection{Gaussian pump beam}

The equations which describe an evolution of correlation in radial spectrum of signal modes in OPA pumped by Gaussian beam can be obtained from Eq. (15). A set of equations for $n$th mode has a form

$$
\frac{\partial B_{1 n}\left(p_{1}, p_{2}\right)}{\partial \xi}=\mathrm{i}\left(p_{1}^{2}-p_{2}^{2}\right) \sqrt{G} B_{1 n}\left(p_{1}, p_{2}\right)
$$

$$
\begin{gathered}
-\mathrm{i}(-1)^{n} G \int_{0}^{\infty} q\left[H_{n}\left(p_{2}, q\right) B_{2 n}\left(p_{1}, q\right)\right. \\
\left.\quad-H_{n}\left(p_{1}, q\right) B_{2 n}^{*}\left(p_{2}, q\right)\right] \mathrm{d} q, \\
\frac{\partial B_{2 n}\left(p_{1}, p_{2}\right)}{\partial \xi}=\mathrm{i}\left(p_{1}^{2}+p_{2}^{2}\right) \sqrt{G} B_{2 n}\left(p_{1}, p_{2}\right) \\
+\mathrm{i}(-1)^{n} G \int_{0}^{\infty} q\left[H_{n}\left(p_{2}, q\right) B_{1 n}\left(p_{1}, q\right)\right. \\
\left.+H_{n}\left(p_{1}, q\right) B_{1,-n}\left(p_{2}, q\right)\right] \mathrm{d} q, \\
\frac{\partial B_{1,-n}\left(p_{1}, p_{2}\right)}{\partial \xi}=\mathrm{i}\left(p_{1}^{2}-p_{2}^{2}\right) \sqrt{G} B_{1,-n}\left(p_{1}, p_{2}\right) \\
-\mathrm{i}(-1)^{n} G \int_{0}^{\infty} q\left[H_{n}\left(p_{2}, q\right) B_{2 n}\left(q, p_{1}\right)\right. \\
\left.-H_{n}\left(p_{1}, q\right) B_{2 n}^{*}\left(q, p_{2}\right)\right] \mathrm{d} q .
\end{gathered}
$$

These equations should be solved with the boundary conditions at $\xi=0$ :

$$
B_{1, n}\left(p_{1}, p_{2}\right)=\frac{D}{2 \pi} \delta\left(p_{1}-p_{2}\right), B_{2 n}\left(p_{1}, p_{2}\right)=0 .
$$

\section{Numerical results}

Equations (24) were solved numerically and evolution of correlation functions $B_{1 n}$ and $B_{2 n}$, as well as variation of spectral intensity $\left\langle\left|S_{n}(p)\right|^{2}\right\rangle=B_{1 n}(p, p)$ and mode intensity $\left\langle\left|F_{n}(r)\right|^{2}\right\rangle$ on propagation length in OPA were analysed.

The intensity of radial spectrum at the output of OPA is shown in Fig. 3 for three signal modes. With a decrease of pump beamwidth $d\left(L_{d} \propto d^{2}\right)$ the parametric gain of vortex modes with $n \neq 0$ becomes smaller in comparison with a fundamental mode. Simultaneously the parametric gain is also decreasing for all modes due to decrease of pump beam power $\left(\propto d^{2}\right)$ : compare with a dotted line in Fig. 3(a), which corresponds to a plane pump wave. For a pump beam with the wavelength $\lambda=0.5 \mu \mathrm{m}$ and radius $d=100 \mu \mathrm{m}$ in the nonlinear crystal (length $1 \mathrm{~cm}$, refraction index $n_{r}=1.5$ ) we find $L_{d} / l=\pi n_{r} d^{2} /(l \lambda) \approx 9.4$. The evolution of normalized correlation function $B_{1 n}$ under propagation in OPA for two signal modes is shown in Fig. 4. The width of correlation function (degree of correlation) increases with a propagation length. Hence the phasing of the components of radial spectrum takes place in OPA pumped by the beam with a finite beamwidth. At rather 

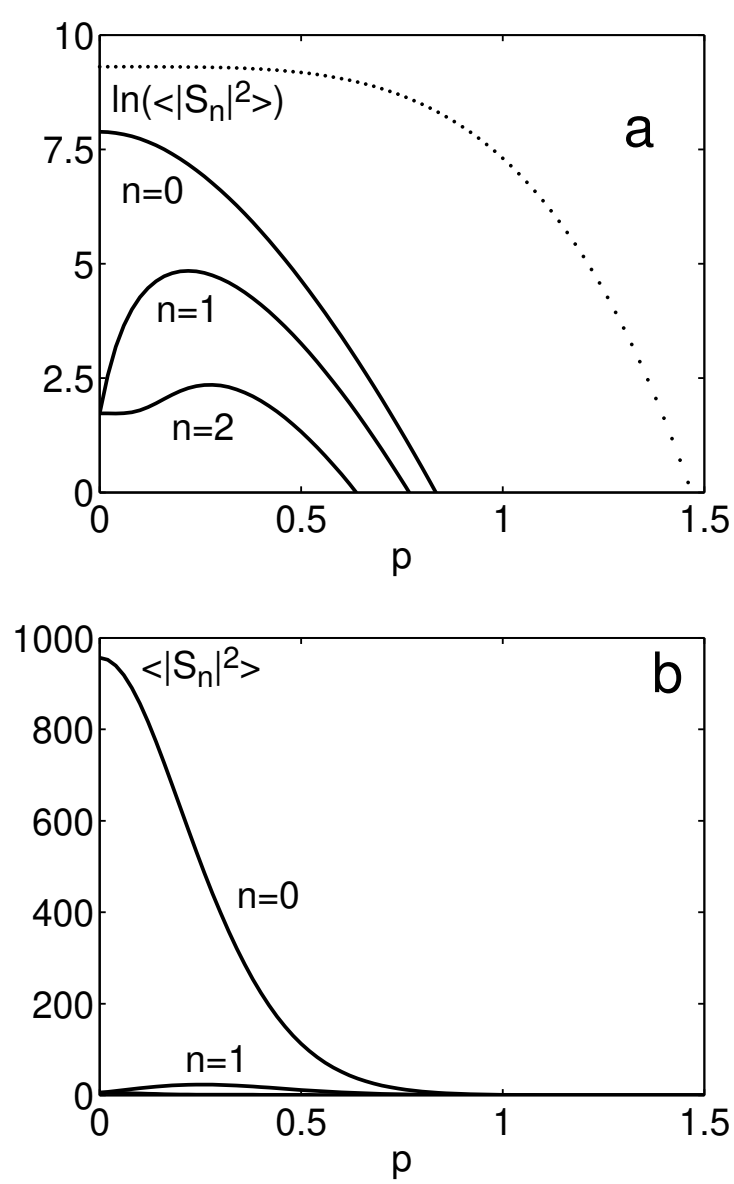

Fig. 3. Normalized to $D /(2 \pi)$ intensity of radial spectrum for three signal modes $(n=0,1,2)$ at the output of OPA. $G=10 . L_{d} / l=$ 20 in (a) and 10 in (b). Dotted line corresponds to plane pump wave.

low parametric gain a degree of correlation in the radial spectrum of modes with $n \neq 0$ is larger for radial frequencies $p \ll 1$, Fig. 4(b). At large parametric gain the maximum of correlation degree is shifted to the larger values of $p$ (Fig. 4(c)) due to the shape of coupling function $H_{n}(p)$, see Fig. 1(b). The degree of correlation in the radial spectrum depends on a pump beamwidth $d$. The normalized correlation function $B_{1 n}$ at the output of OPA is shown in Fig. 5 for two modes at different values of $L_{d} / l$. The distribution of normalized intensity $P_{n}(\rho)$ of two signal modes at the output of OPA is shown in Fig. 6. Hence, the intensity of the mode with a nonzero topological charge is considerably smaller in comparison with intensity of fundamental mode, see Fig. 2. Due to nonuniform profile of pump beam the largest gain in OPA occurs at the axis $(r=0)$ of Gaussian beam. For this reason the modes with the nonzero topological charges are suppressed, and simultaneously the azimuthal correlation is introduced into the noise field under propagation of signal wave in OPA. In this way the phasing of spatial spectrum of amplified sig-
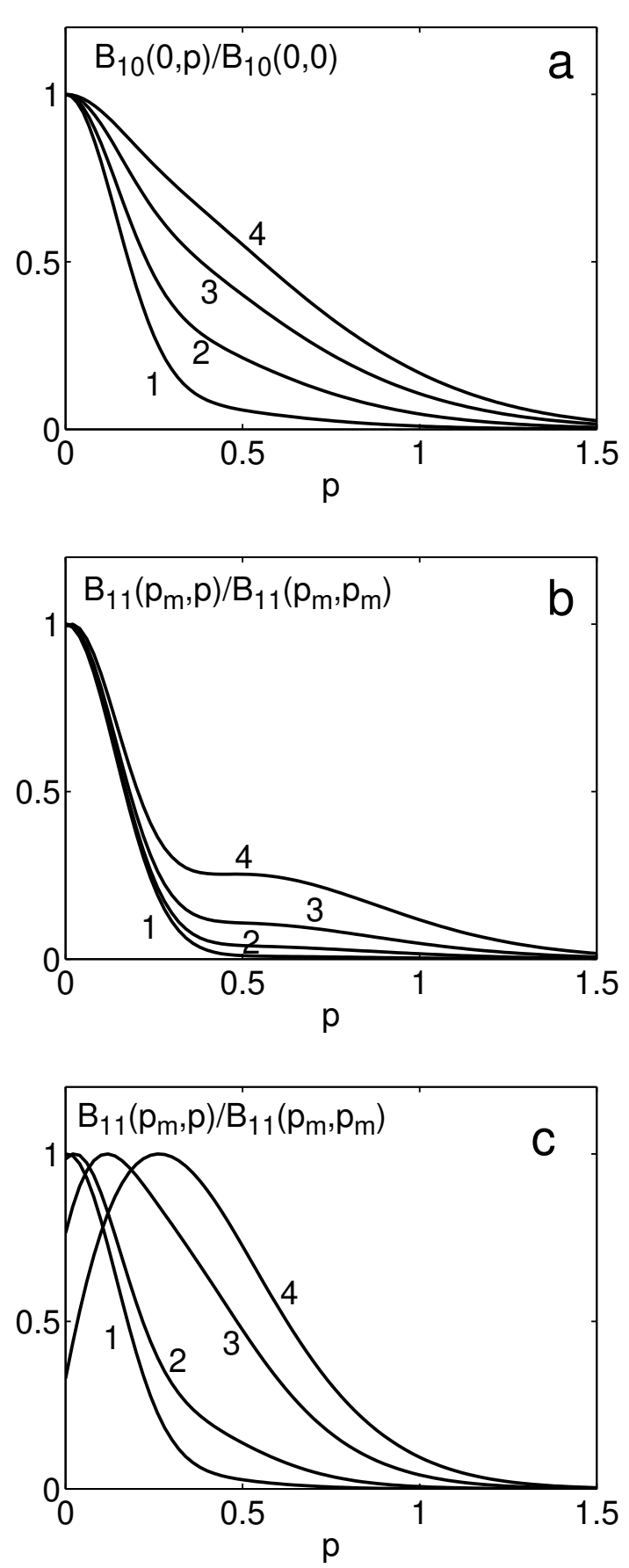

Fig. 4. Evolution of correlation function $B_{1 n}$ under propagation in OPA for two signal modes $(n=0,1)$. $p_{m}$ corresponds to maximum value of $B_{11} . G=10, L_{d} / l=3$ in (a,b), 10 in (c). $\xi: 0.25$ (1), 0.5 (2), 0.75 (3), 1.0 (4).

nal beam from quantum noise level by narrow Gaussian pump beam becomes possible, and the signal beam with a good spatial quality is observed at the output of OPA, as in experiment described in Ref. [3]. 

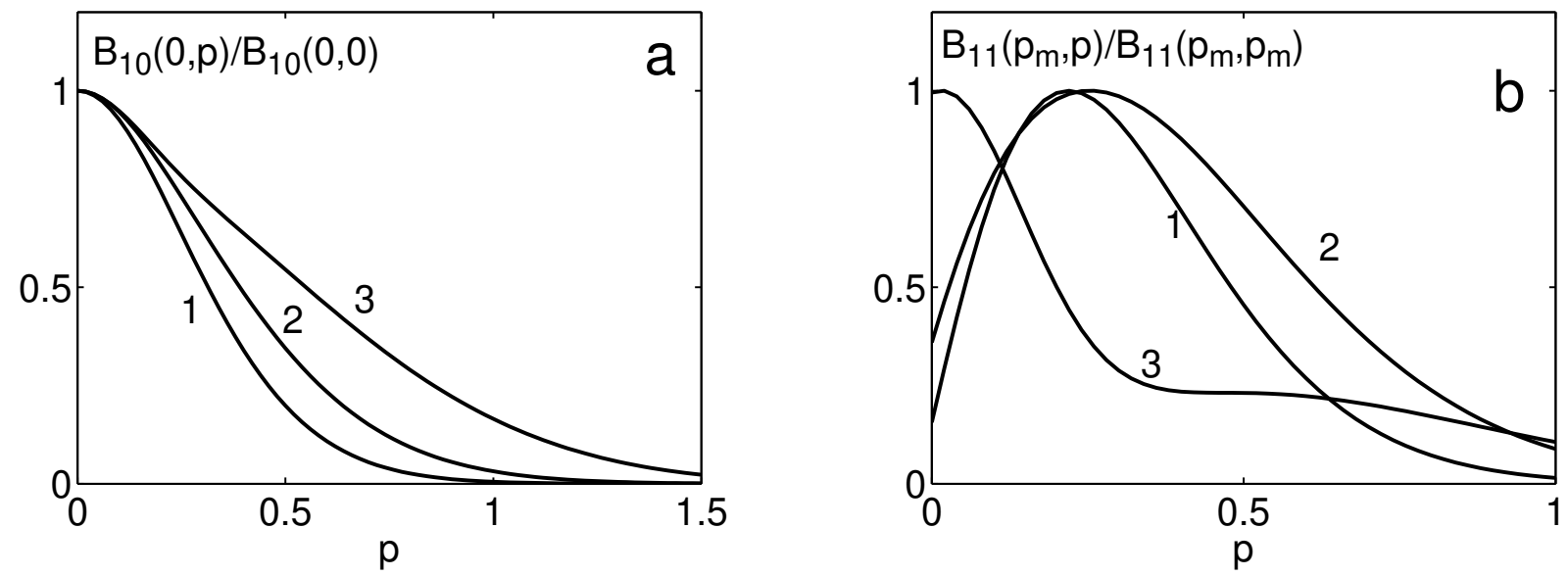

Fig. 5. Correlation functions $B_{1 n}$ at the output of OPA for different values $L_{d} / l$. $G=10 . p_{m}$ corresponds to maximum value of $B_{11} . n=$ 0 in (a), 1 in (b); $L_{d} / l: 20(1), 10(2), 3(3)$.

\section{Conclusions}

It was shown that in cylindrical coordinates a random optical field can be described in classical approach as a superposition of orthogonal coaxial vortex modes with random amplitudes and of various topological charges $n$. It was demonstrated that only vortex modes with opposite topological charges are coupled under parametric amplification, and that essentially simplifies the analysis of correlation in the random optical field.

In OPA pumped by a plane wave the parametric gain of all vortex modes is the same. In contrast to this, the largest parametric gain in OPA pumped by Gaussian beam is observed for fundamental mode $(n=0)$. As a result, with decrease of pump beamwidth a suppression of modes with nonzero topological charges occurs, and at large gain an azimuthal correlation in amplified random optical field appears. Simultaneously, the phasing of the components of radial spectrum takes place. In

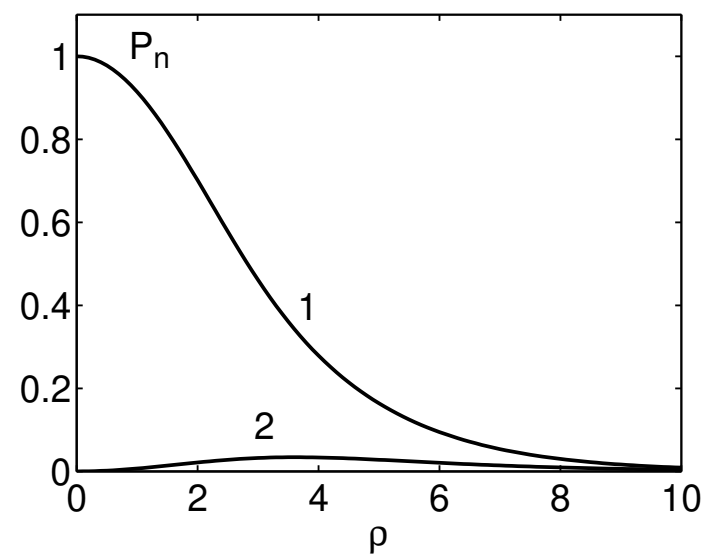

Fig. 6. Distribution of normalized intensity of signal modes at the output of OPA. $G=10, L_{d} / l=20 . n=0$ (1), 1 (2). this way an optical beam of high correlation degree can be obtained under parametric amplification of random optical fields in OPA.

\section{References}

[1] A. Beržanskis, W. Chinaglia, L.A. Lugiato, K.H. Feller, and P. Di Trapani, Spatial structures in optical parametric amplification, Phys. Rev. A 60(2), 1626-1635 (1999).

[2] A. Picozzi and M. Haelterman, Influence of walkoff, dispersion, and diffraction on the coherence of parametric fluorescence, Phys. Rev. E 63(5), 056611 (2001).

[3] P. Di Trapani, G. Valiulis, W. Chinaglia, and A. Andreoni, Two-dimensional spatial solitary waves from traveling-wave parametric amplification of the quantum noise, Phys. Rev. Lett. 80(2), 265-268 (1998).

[4] P. Di Trapani, A. Beržanskis, S. Minardi, S. Sapone, and W. Chinaglia, Observation of optical vortices and $J_{0}$ Bessel-like beams in quantum-noise parametric amplification, Phys. Rev. Lett. 81(23), 5133-5136 (1998).

[5] O. Jedrkiewicz, A. Picozzi, M. Clerici, D. Facio, and P. Di Trapani, Emergence of $X$-shaped spatiotemporal coherence in optical waves, Phys. Rev. Lett. 97(24), 243903 (2006).

[6] O. Jedrkiewicz, M. Clerici, A. Picozzi, D. Facio, and P. Di Trapani, $X$-shaped space-time coherence in optical parametric generation, Phys. Rev. A 76(3), 033823 (2007).

[7] S. Orlov, A. Stabinis, V. Smilgevicius, G. Valiulis, and A. Piskarskas, Parametric excitation of X-waves by downconversion of Bessel beams in nonlinear crystals, Opt. Lett. 32(1), 68-70 (2007).

[8] A. Picozzi, S.-P. Gorza, and M. Haelterman, Role of topological phase-defects in the parametric generation process, Opt. Commun. 281(11), 3196-3200 (2008). 
[9] A. Beržanskis, A. Matijošius, A. Piskarskas, V. Smilgevičius, and A. Stabinis, Conversion of topological charge of optical vortices in a parametric frequency converter, Opt. Commun. 140(4-6), 273-276 (1997).

[10] J. Arlt, K. Dholakia, L. Allen, and M.J. Padgett, Parametric down-conversion for light beams possessing orbital angular momentum, Phys. Rev. A 59(5), 39503952 (1999).

[11] V. Pyragaite, A. Piskarskas, K. Regelskis, V. Smilgevicius, A. Stabinis, and S. Mikalauskas, Parametric down-conversion of higher-order Bessel optical beams in quadratic nonlinear medium, Opt. Commun. 240 (1-3), 191-200 (2004).

[12] M.V. Berry, Disruption of wavefronts: Statistics of dislocations in incoherent Gaussian random waves,
J. Phys. A 11(1), 27-37 (1978).

[13] K. Staliunas, A. Berzanskis, and V. Jarutis, Vortex statistics in optical speckle fields, Opt. Commun. 120(1-2), 23-28 (1995).

[14] M.V. Berry and M.R. Dennis, Phase singularities in isotropic random waves, Proc. R. Soc. A 456(2001), 2059-2079 (2000).

[15] W. Wang, S.G. Hanson, Y. Miyamoto, and M. Takeda, Experimental investigation of local properties and statistics of optical vortices in random wave fields, Phys. Rev. Lett. 94(10), 103902 (2005).

[16] K. O'Holleran, M.R. Dennis, F. Flossmann, and M.J. Padgett, Fractality of light's darkness, Phys. Rev. Lett. 100(5), 053902 (2008).

\title{
PARAMETRINIS TRIUKŠMINIŲ OPTINIŲ LAUKŲ STIPRINIMAS
}

\author{
V. Pyragaitè, A. Stabinis \\ Vilniaus universitetas, Vilnius, Lietuva
}

\section{Santrauka}

Parodyta, kad kvantinio triukšmo sukeltas parametrinis generavimas gali būti aprašytas klasikiniame artinyje kaip ortogonaliu bendraašių sūkurinių modų su atsitiktinèmis amplitudèmis ir įvairiais topologiniais krūviais $n$ parametrinis stiprinimas. Kaupinant
Gauso pluoštu, didžiausias parametrinis stiprinimas yra būdingas pagrindinei modai $(n=0)$, o kitos modos yra slopinamos. Analizuojama įvairių modų radialinio spektro koreliacijos evoliucija vykstant parametriniam stiprinimui. 